\title{
The Control Design of Ship Formation with the Presence of a Leader
}

\author{
Miswanto $^{1}$, I. Pranoto ${ }^{2}$, H. Muhammad ${ }^{3}$, D. Mahayana ${ }^{4}$ \\ ${ }^{1}$ Departement of Mathematics, Airlangga University (UNAIR), Surabaya, Indonesia \\ ${ }^{2}$ Departement of Mathematics, Institut Teknologi Bandung (ITB), Bandung, Indonesia \\ ${ }^{3}$ Aeronautics and Astronautics, Institut Teknologi Bandung (ITB), Bandung, Indonesia \\ ${ }^{4}$ Control System and Computer, Institut Teknologi Bandung (ITB), Bandung, Indonesia
}

\begin{abstract}
Article Info
Article history:

Received Mei 28, 2014

Revised Aug 20, 2014

Accepted Sep 10, 2014

Keyword:

Dynamic System of Ship

Model of Swarm

Numerical Simulation

Pontryagin Maximum Principle

Tracking Error

ABSTRACT

Formation control is an important behavior for multi-agents system (swarm). This paper addresses the optimal tracking control problem for swarm whose agents are ships moving together in a specific geometry formation. We study formation control of the swarm model which consists of three agents and one agent has a role as a leader. The agents of swarm are moving to follow the leader path. First, we design the control of the leader with Pontryagin Maximum Principle. The control of the leader is designed for tracking the desired path. We show that the tracking error of the path of the leader tracing a desired path is sufficiently small. After that, geometry approach is used to design the control of the other. We show that the positioning and the orientation of each agent can be controlled dependent on the leader. The simulation results show to illustrate of this method at the last section of this paper.
\end{abstract}

Copyright (c) 2015 Institute of Advanced Engineering and Science. All rights reserved.

\section{Corresponding Author:}

Miswanto,

Departement of Mathematics,

Faculty of Sciences and Technology,

Airlangga University,

Kampus C Jl. Mulyorejo Surabaya 60115, Indonesia.

Email: miswanto@fst.unair.ac.id

\section{INTRODUCTION}

In recent years, there have been an increasing number of researches on the subject of underactuated vehicle. The example of underactuated vehicle we will focus on is a surface vessel (ship) moving to track a desired path. The tracking problem is a challenging problem in surface vessel. Some researchers have discussed the control design of a surface vessel to track a desired path in [1], [3], [5], [7]. In [1], the authors study the tracking problem of underactuated surface vessel using adaptive control. They design a continuous time-varying tracking controller that forces the position/orientation tracking error to an arbitrarily small neighborhood about zero in the presence of uncertainty in the hydrodynamic damping coefficients. In [3], the authors study the problem of position tracking of underactuated vehicles in both two and three-dimensional spaces. The main contribution is a design methodology to construct a nonlinear tracking controller that yields global stability and exponential convergence of the position tracking error to a neighborhood of the origin that can be made arbitrarily small. Furthermore, the desired trajectory does not need to be a trimming trajectory and can be any sufficiently smooth time-varying bounded curve, including the degenerate case of a constant trajectory (set-point). In [5], the authors study the control design and the tracking problem for a nonlinear underactuated system. They describe how to use backstepping to develop control laws to perform 
trajectory tracking for a nonlinear, underactuated surface vessel. His research extends earlier backstepping designs for underactuated vessels by explaining how to select outputs when generalized forces act on the vessel. In [7], the authors consider tracking control of a surface vessel with only two control inputs. They discuss tracking control of both the position variables and the course angle of the surface vessel. They thus seek to control angle degrees of freedom with only two control inputs variable.

In [10], the author studies the problem of controlling the planar position and orientation of an autonomous surface vessel using two independent thrusters. He shows that although the system is not asymptotically stabilizable to a given equilibrium solution using a time-invariant continuous feedback, it is strongly accessible and small-time locally controllable at any equilibrium and, hence, the system is asymptotically stabilizable to a desired equilibrium using time-invariant discontinuous feedback laws. In [2], the authors consider a linear system with delay in state and control with both matched and unmatched perturbations. They apply the block control technique to design a sliding mode regulator that guarantees asymptotic reference tracking for a class of linear delayed systems with disturbances. This class of systems is those presented in so-called block controllable form with delay. The block control technique is used to derive a sliding manifold on which the motion of the closed-loop system is stable, and the tracking error is zeroed. In [4], an adaptive tracking control problem is studied for a four wheel mobile robot. The authors propose a formulation for the adaptive tracking problem that meets the natural prerequisite such that it reduces to the state feedback tracking problem if the parameters are known. They derive a general methodology for solving their problem.

In [9], the authors study the formation control of swarm whose agents are Dubin's cars. The agents of swarm are moving to track a desired path. They consider the swarm model with presence of a leader. First, they design the control of the leader with tracking error dynamics. The control of the leader is designed for tracking the desired path. After that, geometry approach is used to design the control of the other. In [8], Miswanto et al. study the tracking problem of a swarm model with the presence of a leader by using the least square method. That model is a control system which consists of many agents and one agent has a role as a leader. The control of optimal motion of the leader is obtained by using the least square method. In particular, this control steers the leader to trace a desired path. In [6], Tang et al. study optimal output tracking control (OOTC) problem for a class of bilinear systems with a quadratic performance index using a successive approximation approach (SAA). They develop a design process of the OOTC law based on the SAA for bilinear system.

In this paper we consider formation control of the swarm model whose agents are ships moving together in a specific geometry formation. In this model, one ship has a role as a leader. The control of the leader is designed for tracking the desired path. We show that the tracking error of the path of the leader tracing a desired path is sufficiently small and the distance between the leader ship path and the desired path is preserved. In the next section, the formal problem formulation is described. In section 3, we design the control of the leader ship using Pontryagin Maximum Principle. In section 4, we design the control of each agent follower using geometry approach. In section 5, we show numerical simulations to illustrate our results.

\section{PROBLEM FORMULATION}

In this section, we introduce the dynamic system of the model ship as shown in Figure 1.

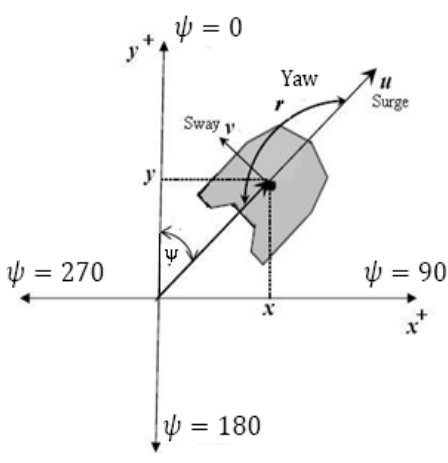

Figure 1. The model ship

IJRA Vol. 4, No. 1, March 2015 : 53 -62 
In this paper, the dynamics system of ship is taked from Tzeng and Chen model. They only discuss one ship and in this paper discuss for three ships which described as:

$$
\begin{aligned}
\dot{x}_{i}(t) & =u_{i}(\mathrm{t}) \cos \left(\psi_{i}(t)\right)-v_{i}(\mathrm{t}) \sin \left(\psi_{i}(t)\right) \\
\dot{y}_{i}(t) & =u_{i}(\mathrm{t}) \sin \left(\psi_{i}(t)\right)+v_{i}(\mathrm{t}) \cos \left(\psi_{i}(t)\right) \\
\dot{u}_{i}(t) & =0 \\
\dot{v}_{i}(t) & =0 \\
\dot{\psi}_{i}(t) & =r_{i}(t) \\
\dot{r}_{i}(t) & =-0.0562 r_{i}(t)+\delta_{i}(t)
\end{aligned} \quad i=1,2,3
$$

Where $\left(x_{i}(t), y_{i}(t)\right) \in R^{2}$ represents the position of the $i$-th ship, and $\psi_{i}(t) \in[0,2 \pi]$ represents the orientation of the $i$-th ship. $u_{i}(t)$ are velocities in surge of the $i$-th ship, $v_{i}(t)$ are velocities in sway of the $i$-th ship, $r_{i}(t)$ are yaw rate of the $i$-th ship and $\delta_{i}(t)$ are ruder angle of the $i$-th ship. In this paper, the desired path $(\gamma)$ that would be tracked by the leader ship is obtained using calculus variational method. The path is denoted by $\gamma(t)=\left[\gamma_{x}(t), \gamma_{y}(t)\right]^{T}$.

In this paper, there are two problems which will be discussed. First, we design the control of the leader ship for tracking the desired path by Pontryagin Maximum Principle. Furthermore, we design the control of the other agents by geometry approach to follow the leader's path with a certain distance.

\section{THE CONTROL DESIGN OF THE LEADER SHIP}

We consider a model of the leader ship, such as (1). We design the control of the leader ship by Pontryagin Maximum Principle for minimizing the tracking error in order to keep the position of the leader ship close to the desired path. We define a tracking error $e(t)$ as the difference between the actual leader ship path and the desired path

$$
e(t)=\left[x_{1}-\gamma_{x}, y_{1}-\gamma_{y}\right]^{T}
$$

Thus, $\boldsymbol{e}(t)$ lives in $R^{2}$ for every $t$. The original problem is translated to the following optimal control problem. We search for $\left(\delta_{1}(t)\right)$ that makes the following functional minimized,

$$
J=\frac{1}{2} \int_{0}^{T}\left(\delta_{1}^{2}+k\|e\|^{2}\right) d t
$$

The term $\delta_{1}^{2}$ represents the total cost of the control used by the leader ship and $k\|e\|^{2}$ represents the total error. In this paper, the value of the constant $k$ is restricted to $k=1$. Now, the Hamiltonian function of the system is

$$
H=p_{1} \dot{x}_{1}+p_{2} \dot{y}_{1}+p_{3} \dot{u}_{1}+p_{4} \dot{v}_{1}+p_{5} \dot{\psi}_{1}+p_{6} \dot{r}_{1}+\frac{1}{2} p_{0}\left(\delta_{1}^{2}+k\|e\|^{2}\right) .
$$

Using this function, we build the Hamiltonian system: 


$$
\begin{aligned}
& \frac{\partial H}{\partial p_{1}}=\dot{x}_{1}(t)=u_{1}(\mathrm{t}) \cos \left(\psi_{1}(t)\right)-v_{1}(\mathrm{t}) \sin \left(\psi_{1}(t)\right) \\
& \frac{\partial H}{\partial p_{2}}=\dot{y}_{1}(t)=u_{1}(\mathrm{t}) \sin \left(\psi_{1}(t)\right)+v_{1}(\mathrm{t}) \cos \left(\psi_{1}(t)\right) \\
& \frac{\partial H}{\partial p_{3}}=\dot{u}_{1}=0 \\
& \frac{\partial H}{\partial p_{4}}=\dot{v}_{1}=0 \\
& \frac{\partial H}{\partial p_{5}}=\dot{\psi}(t)=r_{1}(t) \\
& \frac{\partial H}{\partial p_{6}}=\dot{r}_{1}(t)=-0.0562 r_{1}(t)+\delta_{1}(t) \\
& \frac{\partial H}{\partial x_{1}}=-\dot{p}_{1}(t)=p_{0}\left(x_{1}(t)-\gamma_{x}(t)\right) \\
& \frac{\partial H}{\partial y_{1}}=-\dot{p}_{2}(t)=p_{0}\left(y_{1}(t)-\gamma_{y}(t)\right) \\
& \frac{\partial H}{\partial u_{1}}=-\dot{p}_{3}(t)=p_{1}(t) \cos \left(\psi_{1}(t)\right)+p_{2}(t) \sin \left(\psi_{1}(t)\right) \\
& \frac{\partial H}{\partial v_{1}}=-\dot{p}_{4}(t)=-p_{1}(t) \sin \left(\psi_{1}(t)\right)+p_{2}(t) \cos \left(\psi_{1}(t)\right) \\
& \frac{\partial H}{\partial \psi_{1}}=-\dot{p}_{5}(t)=-p_{1}(t)\left(u_{1}(t) r_{1}(t) \sin \left(\psi_{1}(t)\right)+v_{1}(t) r_{1}(t) \cos \left(\psi_{1}(t)\right)\right) \\
& +p_{2}(t)\left(u_{1}(t) r_{1}(t) \cos \left(\psi_{1}(t)\right)-v_{1}(t) r_{1}(t) \sin \left(\psi_{1}(t)\right)\right) \\
& \frac{\partial H}{\partial r_{1}}=-\dot{p}_{6}(t)=p_{5}(t)-0.0562 p_{6}(t)
\end{aligned}
$$

By the Pontryagin Maximum Principle, the value of $H$ must be optimized with respect to the control $\left(\delta_{1}\right)$. Thus $\frac{\partial H}{\partial \delta_{1}}=p_{6}(t)+p_{0}(t) \delta_{1}(t)=0$, since $p_{0}$ must be constant and negative, without loss of generality, we let $p_{0}=-1$. Thus, we obtain the control $\delta_{1}(t)=p_{6}(t)$. Then, this control is substituted in (5). Thus, we obtain a system of differential equations: 


$$
\begin{aligned}
& \dot{x}_{1}(t)=u_{1}(\mathrm{t}) \cos \left(\psi_{1}(t)\right)-v_{1}(\mathrm{t}) \sin \left(\psi_{1}(t)\right) \\
& \dot{y}_{1}(t)=u_{1}(\mathrm{t}) \sin \left(\psi_{1}(t)\right)+v_{1}(\mathrm{t}) \cos \left(\psi_{1}(t)\right) \\
& \dot{u}_{1}(t)=0 \\
& \dot{v}_{1}(t)=0 \\
& \dot{\psi}_{1}(t)=r_{1}(t) \\
& \dot{r}_{1}(t)=-0.0562 r_{1}(t)+p_{6}(t) \\
& \dot{p}_{1}(t)= k\left(x_{1}(t)-\gamma_{x}(t)\right) \\
& \dot{p}_{2}(t)=k\left(y_{1}(t)-\gamma_{y}(t)\right) \\
& \dot{p}_{3}(t)=-p_{1}(t) \cos \left(\psi_{1}(t)\right)-p_{2}(t) \sin \left(\psi_{1}(t)\right) \\
& \dot{p}_{4}(t)=p_{1}(t) \sin \left(\psi_{1}(t)\right)-p_{2}(t) \cos \left(\psi_{1}(t)\right) \\
& \dot{p}_{5}(t)=p_{1}(t)\left(u_{1}(t) r_{1}(t) \sin \left(\psi_{1}(t)\right)+v_{1}(t) r_{1}(t) \cos \left(\psi_{1}(t)\right)\right) \\
& \quad-p_{2}(t)\left(u_{1}(t) r_{1}(t) \cos \left(\psi_{1}(t)\right)-v_{1}(t) r_{1}(t) \sin \left(\psi_{1}(t)\right)\right) \\
& \dot{p}_{6}(t)=0.0562 p_{6}(t)-p_{5}(t)
\end{aligned}
$$

Initial and final conditions of the state variables $\left[x_{1}, y_{1}, u_{1}, v_{1}, \psi_{1}, r_{1}\right]^{T}$ are known. However, the costate equations $\left[p_{i} ; i=1,2, \ldots 6\right]^{T}$ does not have initial condition. So, the solution of the system of differential equation is difficult to obtain. This paper proposes the steepest gadient descent method. This method is used to approximate the initial condition of the costate variables in the system of differential equation. First, the initial value of state variables are given by $x_{i}(0)=x_{i 0} ; i=1,2,3,4,5,6$ and the initial value of costate variables are arbitrarily guessed by $p_{i}(0)=q_{i 0} ; i=1,2,3,4,5,6$. The values are used to solve the system of differential equation. Next, we calculate $F\left(q_{10}, q_{20}, q_{30}, q_{40}, q_{50}, q_{60}\right)=\sum_{i=1}^{6}\left\|x_{i}(T)-x_{i T}\right\|^{2}$, where $x_{i}(T)$ is obtained from the solution of the system of differential equation and $x_{i T}$ is the final conditions of the state variables. Afterwards, we determine the value of the new $\left(q_{10}, q_{20}, q_{30}, q_{40}, q_{50}, q_{60}\right)$ by using the steepest gradient descent method, such as in Tjahjana [7]. The value is used to make the new $F\left(q_{10}, q_{20}, q_{30}, q_{40}, q_{50}, q_{60}\right)$ less than the old $F\left(q_{10}, q_{20}, q_{30}, q_{40}, q_{50}, q_{60}\right)$. The process is done repeatedly until the value of $F\left(q_{10}, q_{20}, q_{30}, q_{40}, q_{50}, q_{60}\right)$ is small enough.

\section{THE CONTROL DESIGN OF THE FOLLOWING AGENTS}

In this section, we design the control of the follower using geometry approach. Figure 2 shows the position of the three ships. Where $d 1$ and $d 2$ are the distance of the follower ship to the leader ship. $\theta_{2}$ and $\theta_{3}$ are the orientation of the follower ship to position of the leader ship. In this paper, $\theta_{2}$ and $\theta_{3}$ are assumed to be constant.

\subsection{The Control Design of The First Follower Ship}

From the Figure 2, we have

$$
\begin{aligned}
& x_{1}(t)-x_{2}(t)=d_{1} \sin \left(\psi_{2}(t)-\theta_{2}\right) \\
& y_{1}(t)-y_{2}(t)=d_{1} \cos \left(\psi_{2}(t)-\theta_{2}\right)
\end{aligned}
$$




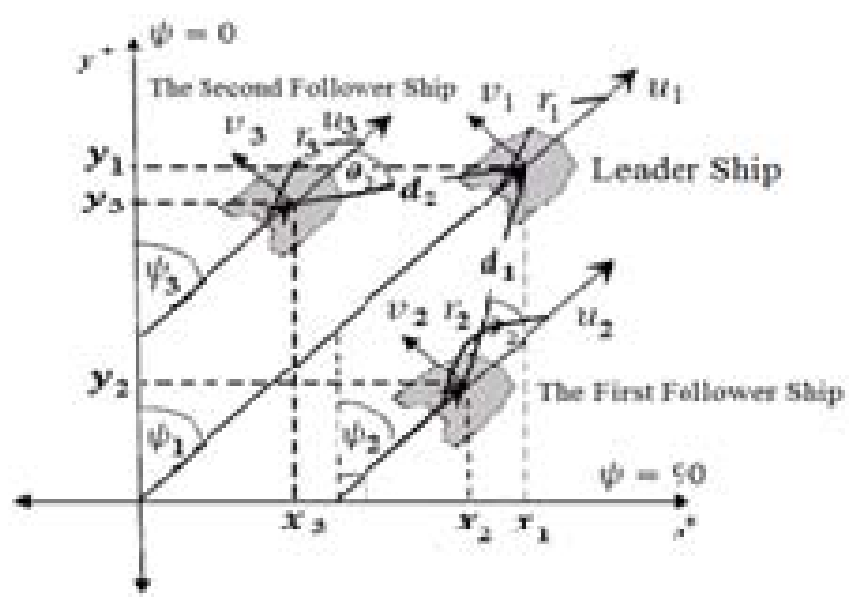

Figure 2. The Position of Three Ships

Differentiating the equations above with respect to time, we obtain

$$
\begin{aligned}
& \dot{x}_{1}(t)-\dot{x}_{2}(t)=d_{1} \cos \left(\psi_{2}(t)-\theta_{2}\right)\left(\dot{\psi}_{2}(t)-\dot{\theta}_{2}\right) \\
& \dot{y}_{1}(t)-\dot{y}_{2}(t)=-d_{1} \sin \left(\psi_{2}(t)-\theta_{2}\right)\left(\dot{\psi}_{2}(t)-\dot{\theta}_{2}\right),
\end{aligned}
$$

because $\theta_{2}$ assumed to be constant, then the system of equations (8) above can be written as

$$
\begin{aligned}
& \dot{x}_{1}(t)-\dot{x}_{2}(t)=d_{1} r_{2}(t) \cos \left(\psi_{2}(t)-\theta_{2}\right) \\
& \dot{y}_{1}(t)-\dot{y}_{2}(t)=-d_{1} r_{2}(t) \sin \left(\psi_{2}(t)-\theta_{2}\right)
\end{aligned}
$$

Thus, we obtain

$$
\begin{aligned}
& \left(\dot{x}_{1}(t)-\dot{x}_{2}(t)\right) \cos \left(\psi_{2}(t)-\theta_{2}\right) \\
& \quad-\left(\dot{y}_{1}(t)-\dot{y}_{2}(t)\right) \sin \left(\psi_{2}(t)-\theta_{2}\right)=d_{1} r_{2}(t)
\end{aligned}
$$

Differentiating the equations (10) above with respect to time, we obtain,

$$
\begin{gathered}
\left(\ddot{x}_{1}(t)-\ddot{x}_{2}(t)\right) \cos \left(\psi_{2}(t)-\theta_{2}\right)-r_{2}(t)\left(\dot{x}_{1}(t)-\dot{x}_{2}(t)\right) \sin \left(\psi_{2}(t)-\theta_{2}\right) \\
-\left(\ddot{y}_{1}(t)-\ddot{y}_{2}(t)\right) \sin \left(\psi_{2}(t)-\theta_{2}\right)-r_{2}(t)\left(\dot{y}_{1}(t)-\dot{y}_{2}(t)\right) \cos \left(\psi_{2}(t)-\theta_{2}\right) \\
=d_{1}\left(-0.0562 r_{2}(t)+\delta_{2}(t)\right)
\end{gathered}
$$

Substituting system (1) for $\mathrm{i}=2$ in the equation (11), one obtain

$$
\begin{array}{r}
\delta_{2}(t)=0.0562 r_{2}(t) \\
+\frac{2 r_{2}(t)\left(u_{2}(t) \sin \left(\psi_{2}(t)+\psi_{2}(t)-\theta_{2}\right)+v_{2}(t) \cos \left(\psi_{2}(t)+\psi_{2}(t)-\theta_{2}\right)\right)}{d_{1}} \\
\quad-\frac{r_{1}(t)\left(u_{1}(t) \sin \left(\psi_{1}(t)+\psi_{2}(t)-\theta_{2}\right)+v_{1}(t) \cos \left(\psi_{1}(t)+\psi_{2}(t)-\theta_{2}\right)\right)}{d_{1}} \\
-\frac{r_{2}(t)\left(u_{1}(t) \sin \left(\psi_{1}(t)+\psi_{2}(t)-\theta_{2}\right)+v_{1}(t) \cos \left(\psi_{1}(t)+\psi_{2}(t)-\theta_{2}\right)\right)}{d_{1}}
\end{array}
$$

Then, this control $\left(\delta_{2}\right)$ is substituted to the system (1) with $i=2$. Thus, we obtain a system of the differential equations of the first follower 


$$
\begin{aligned}
\dot{x}_{2}(t)= & u_{2}(\mathrm{t}) \cos \left(\psi_{2}(t)\right)-v_{2}(\mathrm{t}) \sin \left(\psi_{2}(t)\right) \\
\dot{y}_{2}(t)= & u_{2}(\mathrm{t}) \sin \left(\psi_{2}(t)\right)+v_{2}(\mathrm{t}) \cos \left(\psi_{2}(t)\right) \\
\dot{u}_{2}(t)= & 0 \\
\dot{v}_{2}(t)= & 0 \\
\dot{\psi}_{2}(t)= & r_{2}(t) \\
\dot{r}_{2}(t)= & \frac{2 r_{2}(t)\left(u_{2}(t) \sin \left(2 \psi_{2}(t)-\theta_{2}\right)+v_{2}(t) \cos \left(2 \psi_{2}(t)-\theta_{2}\right)\right)}{d_{1}} \\
& -\frac{r_{1}(t)\left(u_{1}(t) \sin \left(\psi_{1}(t)+\psi_{2}(t)-\theta_{2}\right)+v_{1}(t) \cos \left(\psi_{1}(t)+\psi_{2}(t)-\theta_{2}\right)\right)}{d_{1}} \\
& -\frac{r_{2}(t)\left(u_{1}(t) \sin \left(\psi_{1}(t)+\psi_{2}(t)-\theta_{2}\right)+v_{1}(t) \cos \left(\psi_{1}(t)+\psi_{2}(t)-\theta_{2}\right)\right)}{d_{1}}
\end{aligned}
$$

\subsection{The Control Design of The Second Follower}

From the figure 2, we have

$$
\begin{aligned}
& x_{1}(t)-x_{3}(t)=d_{2} \sin \left(\psi_{3}(t)+\theta_{3}\right) \\
& y_{1}(t)-y_{3}(t)=d_{2} \cos \left(\psi_{3}(t)+\theta_{3}\right)
\end{aligned}
$$

Using similar steps such as in 4.1., one may design the control of the second follower

$$
\begin{gathered}
\delta_{3}(t)=0.0562 r_{3}(t) \\
+\frac{2 r_{3}(t)\left(u_{3}(t) \sin \left(2 \psi_{3}(t)+\theta_{3}\right)+v_{3}(t) \cos \left(2 \psi_{3}(t)+\theta_{3}\right)\right)}{d_{2}} \\
-\frac{r_{1}(t)\left(u_{1}(t) \sin \left(\psi_{1}(t)+\psi_{3}(t)+\theta_{3}\right)+v_{1}(t) \cos \left(\psi_{1}(t)+\psi_{3}(t)+\theta_{3}\right)\right)}{d_{2}} \\
-\frac{r_{3}(t)\left(u_{1}(t) \sin \left(\psi_{1}(t)+\psi_{3}(t)+\theta_{3}\right)+v_{1}(t) \cos \left(\psi_{1}(t)+\psi_{3}(t)+\theta_{3}\right)\right)}{d_{2}}
\end{gathered}
$$

Then, this control $\left(\delta_{3}\right)$ is substituted to the system (1) with $i=3$. Thus, we obtain a system of the differential equations of the second follower:

$$
\begin{aligned}
\dot{x}_{3}(t) & =u_{3}(\mathrm{t}) \cos \left(\psi_{3}(t)\right)-v_{3}(\mathrm{t}) \sin \left(\psi_{3}(t)\right) \\
\dot{y}_{3}(t) & =u_{3}(\mathrm{t}) \sin \left(\psi_{3}(t)\right)+v_{3}(\mathrm{t}) \cos \left(\psi_{3}(t)\right) \\
\dot{u}_{3}(t) & =0 \\
\dot{v}_{3}(t) & =0 \\
\dot{\psi}_{3}(t) & =r_{3}(t) \\
\dot{r}_{3}(t) & =\frac{2 r_{3}(t)\left(u_{3}(t) \sin \left(2 \psi_{3}(t)+\theta_{3}\right)+v_{3}(t) \cos \left(2 \psi_{3}(t)+\theta_{3}\right)\right)}{d_{2}} \\
& -\frac{r_{1}(t)\left(u_{1}(t) \sin \left(\psi_{1}(t)+\psi_{3}(t)+\theta_{3}\right)+v_{1}(t) \cos \left(\psi_{1}(t)+\psi_{3}(t)+\theta_{3}\right)\right)}{d_{2}} \\
& -\frac{r_{3}(t)\left(u_{1}(t) \sin \left(\psi_{1}(t)+\psi_{3}(t)+\theta_{3}\right)+v_{1}(t) \cos \left(\psi_{1}(t)+\psi_{3}(t)+\theta_{3}\right)\right)}{d_{2}}
\end{aligned}
$$

\section{NUMERICAL SOMULATION}

In this section, some numerical simulations to illustrate the system (6), (13) and (16) are reported. The control design of the leader ship used the Pontryagin Maximum Principle for optimal control problems. To approach the initial value of the co-state variables used steepest gradient descent method. Furthermore, the control design of the follower ship used geometry approach (Miswanto, et al., 2012). First of all given a desired path traced by the leader ship. The Start position and end position of the desired path is $\gamma(0)=$ $(0,10)$ dan $\gamma(20)=(2.78,143.14)$. The desired path is written as follows: 


$$
\begin{aligned}
& \gamma_{x}(t)=\frac{10}{3} \cos (0.15 t)+\frac{200}{3} \sin (0.15 t)-\frac{10}{3} \\
& \gamma_{y}(t)=\frac{10}{3} \sin (0.15 t)-\frac{200}{3} \cos (0.15 t)+\frac{230}{3}
\end{aligned}
$$

The problem in this section is to design the control for three ships, so the three ship move to follow the desired path from the initial position ( $t=0$ seconds) to the end ( $t=20$ seconds). First of all is designed motion control of the leader ship for track a desired path. Design control of the leader ship used the Pontriagyn Maximum Principle. Table 1 below shows start position and end position of the leader ship. The leader ship is expected to maneuver tracing this path as close as possible.

Table 1. Start position ( $t=0$ second) and end position ( $t=20$ second) of the leader ship.

\begin{tabular}{cccccccccccc}
\hline$x(0)$ & $y(0)$ & $u(0)$ & $v(0)$ & $\psi(0)$ & $r(0)$ & $x(20)$ & $y(20)$ & $u(20)$ & $v(20)$ & $\psi(20)$ & $r(20)$ \\
$\mathrm{m}$ & $\mathrm{m}$ & $\frac{m}{d e t}$ & $\frac{m}{d e t}$ & $\operatorname{deg}$ & $\frac{d e g}{d e t}$ & $\mathrm{~m}$ & $\mathrm{~m}$ & $\frac{m}{d e t}$ & $\frac{m}{d e t}$ & $\operatorname{deg}$ & $\frac{\operatorname{deg}}{\operatorname{det}}$ \\
\hline 0 & 10 & 10 & 0.5 & 90 & 0 & 2.78 & 143.14 & 10 & 0.5 & -90 & -9 \\
\hline
\end{tabular}

The desired path in equation (17) is substituted in (6) with the parameter $k=1$. As explained above, the initial value of the co-state variable approximated by the method of steepest gradient descent. Fig. 3 below shows the trajectory of the leader ship tracing the desired path by using the method.

From Figure 3 above shows that the trajectory of the leader ship (solidline) can track the desired path (circle) from the strart position $t=0$ seconds to the end position $t=20$ seconds with a small enough distance. This means that the leader ship can move from one area (the starting position) to another area (final position), hope of the results of numerical simulations are the first follower ship and the second follower ship also move to follow the trajectory of the leader ship from the start position ( $t=0$ seconds) to the end position ( $t=20$ seconds). Trajectory error between the trajectories of the leader ship with desired path can be seen in Figure 4.

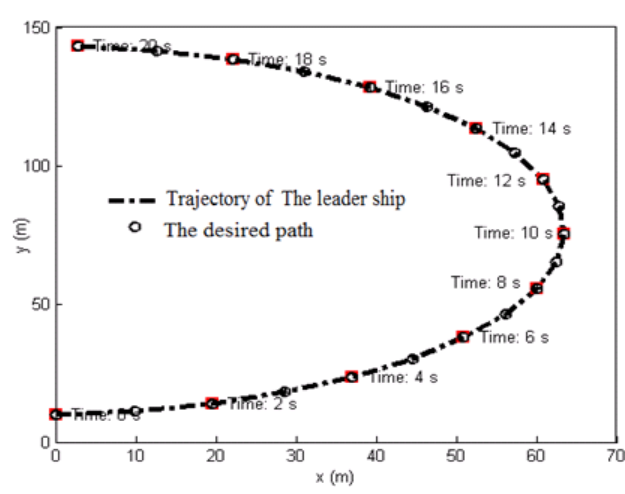

Figure 3. Trajectory of the leader ship and the desired path.
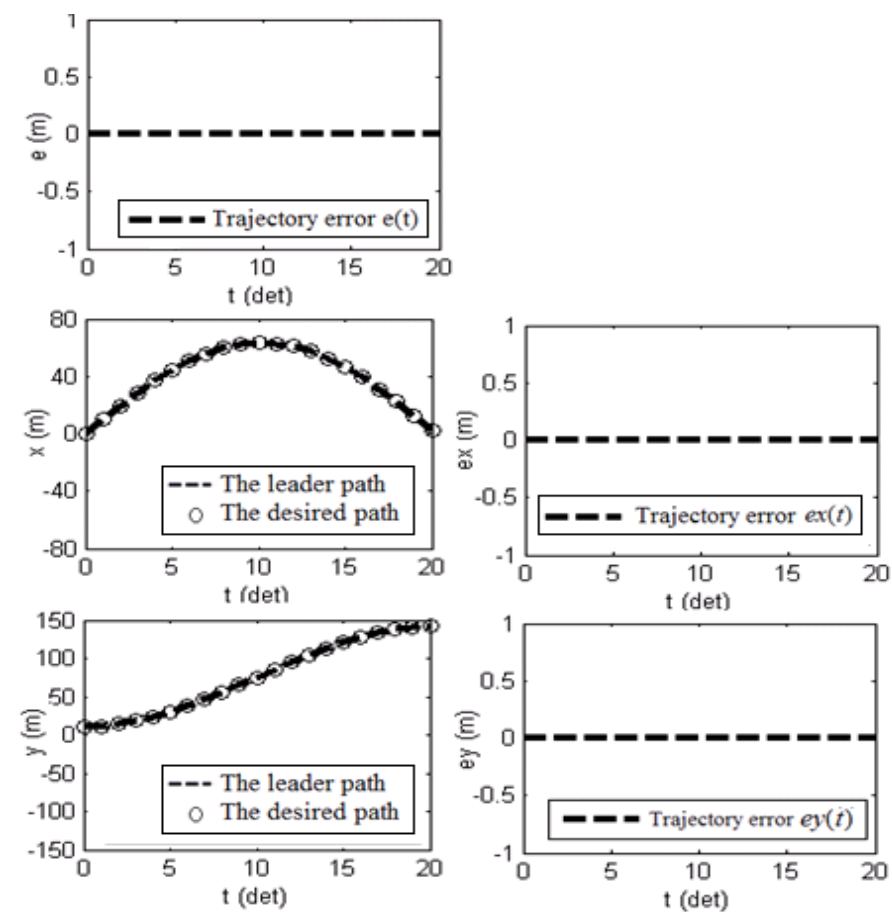

Figure 4. Trajectory Error between the trajectory of the leader ship and desired path 
Next, we show the numerical simulations to illustrate model (1) in the two dimensional space. The movement of the two agents are described by the systems (13) and (16) and the leader is described by the system (6). Distance $\left(d_{1}\right)$ between the initial position of the first follower ship to the initial position of leader ship is 50 $\mathrm{m}$ and the distance $\left(d_{2}\right)$ between the initial position of the second follower ship to initial position of the leader ship is $50 \mathrm{~m}$. Orientation angle $\left(\theta_{2}\right)$ of the first follower ship and second follower ship $\left(\theta_{3}\right)$ to the leader ship is 36 degrees and 10 degrees. In this simulation, velocity of three ship is assumed to equal. The initial conditions of the three ships are given as follows.

Tablel 2. Initial position of three ship

\begin{tabular}{ccccccc}
\hline \multirow{2}{*}{ Kapal } & $\begin{array}{c}x(0) \\
\mathrm{m}\end{array}$ & $\begin{array}{c}y(0) \\
\mathrm{m}\end{array}$ & $\begin{array}{c}u(0) \\
\frac{m}{d e t}\end{array}$ & $\begin{array}{c}v(0) \\
\frac{m}{d e t}\end{array}$ & $\begin{array}{c}\psi(0) \\
\text { Deg }\end{array}$ & $\begin{array}{c}r(0) \\
\text { deg }\end{array}$ \\
\hline Master & 0 & 10 & 10 & 0.5 & 90 & 0 \\
Slave I & 40 & -20 & 10 & 0.5 & 90 & 0 \\
Slave II & -40 & -20 & 10 & 0.5 & 90 & 0 \\
\hline
\end{tabular}

Next, we show the trajectory of the model (1) for three ship. Trajectory of the three ship can be seen in Figure 4.

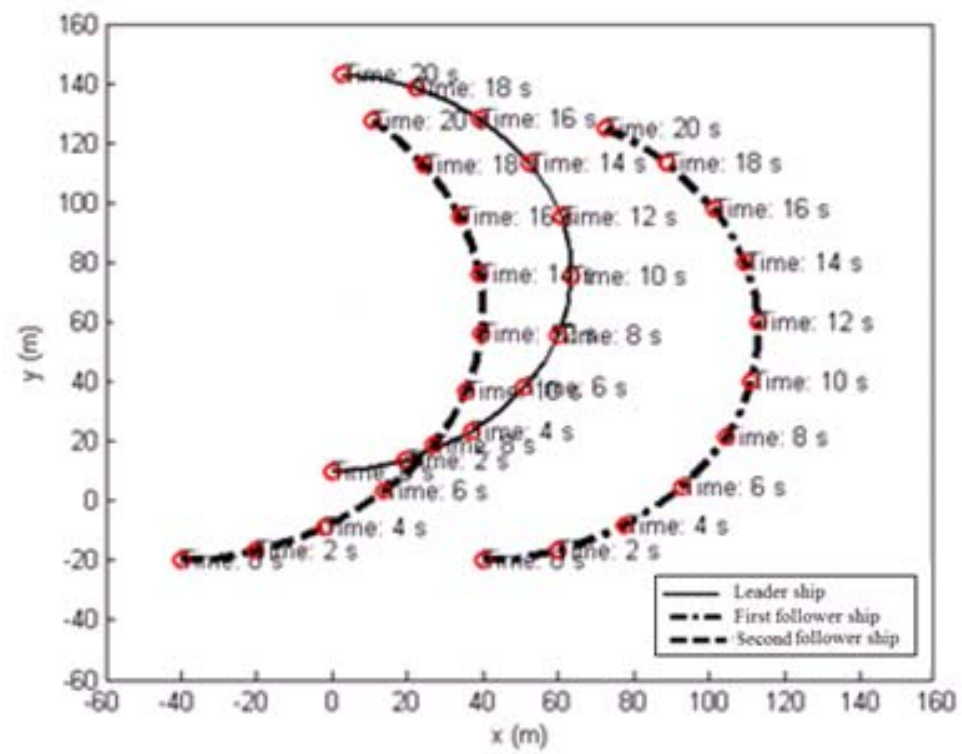

Figure 5. Trajectory of three ship formation

The results of numerical simulations in Figure 4 above shows that two follower ships can follow the trajectory of the leader ship moves from the initial position ( $t=0$ seconds) to end position ( $t=20$ seconds). From Figure 4, it can be seen that the triangle formation of three ship are preserved with the leader positions on the pointing part, but the measurement of the triangle is not preserved. The measurement of the triangle formation is smaller. We suspect that this is caused by the attraction function that is too strong.

\section{CONCLUSION}

A From the numerical simulation results above, it can be seen that the tracking error of the path of the leader ship tracing a desired path is sufficiently small and the distance between the path of leader ship and the desired path is preserved. A geometry approach for formation control of a group of ship is investigated in this paper. The simulation on three ship formation demonstrates that the proposed method is effective and feasible. In the future works, we will discuss the movement control of model swarm consisting of several ships with a specific geometry formation. 


\section{ACKNOWLEDGEMENTS}

Authors would like to thank the my institution (Airlangga University) for financial supporting this research. The authors would also like thanks Directorate General of Higher Education Department of National Education, Indonesia. This research was supported by Unggulan Perguruan Tinggi (UPT) grant under contract number 7673/UN3/KR/2013, 2 May 2013.

\section{REFERENCES}

[1] A. Behal, D. M. Dawson, B. Xian, and P. Setlur, Adaptive Tracking Control of Underactuated Surface Vessels, Proceedings of the IEEE International Conference on Control Applications, 2001, Mexico City, Mexico.

[2] A. G. Loukianov, B. C. Toledo, J. E. Hernandez, E. N. Perez, On the Problem of Tracking for a Class of Linear Systems with Delays and Sliding Modes, International Journal of Control, vol. 76, no. 9, pp. 942 — 958, 2002..

[3] A.P. Aguir and J.P. Hespanha, Position Tracking of Underactuated Vehicles, Proceedings of the American Control Conference, pp. 1988-1993, June 2003, Denver, Colorado.

[4] C.Y. Tzeng and J.F. Chen, Fundamental Properties of Linear Ship Steering dynamic models, Journal of Marine Science and Technology, 1999, Vol. 7, No. 2, pp. 79-88.

[5] E. Lefeber And H. Nijmeijer, Adaptive Tracking Control of Nonholonomic Systems: an example, Proceedings of the $38^{\text {th }}$ Conference on Decision \& Control, Phoenix, December 1999, Arizona USA.

[6] G. J. Toussaint, T. Basar, F. Bullo, Tracking for Nonlinear Underactuated Surface Vessels with Generalized Forces, IEEE Conf. on Control Applications, Anchorage, 2000, Alaska.

[7] G.Y. Tang, Y.D. Zhao and Hui Ma, Optimal Output Tracking Control for Bilinear Systems, Transactions of the Institute of Measurement and Control, 2006, 28, 4, pp. 387-397.

[8] K.Y. Pettersen and H. Nijmeijer, Tracking Control of an Underactuated Surface Vessel, Proceedings of the $37^{\text {th }}$ Conference on Decision \& Control, 1998, FL, USA.

[9] Miswanto, I. Pranoto, and H. Muhammad, A Model of Swarm Movement with The Presence of A Leader, Proceeding, International Conference on Mathematics and Natural Sciences, pp. 740-742, ITB, 2006, Bandung.

[10] Miswanto, I. Pranoto, J. Naiborhu, S. Achmadi, Formation Control of Multiple Dubin's Car System with Geometric Approach, IOSR Journal of Mathematics (IOSRJM), Volume 1, Issue 6 (July-Aug 2012), PP 16-20, 2012.

[11] M. Reyhanoglu, Exponential Stabilization of an Underactuated Autonomous Surface Vessel, Automatica, vol. 33, no. 12, pp. 2249-2254, 1997. 